\title{
The prefrontal cortex and flexible behavior
}

\author{
Helen Barbas $^{1,2}$ and Basilis Zikopoulos ${ }^{1}$ \\ Basilis Zikopoulos: zikopoul@bu.edu \\ ${ }^{1}$ Department of Health Sciences, Boston University, Boston, MA \\ ${ }^{2}$ Program in Neuroscience, Boston University, Boston, MA
}

\begin{abstract}
The prefrontal cortex in primates guides behavior by selecting relevant stimuli for the task at hand, mediated through excitatory bidirectional pathways with structures associated with sensory processing, memory and emotions. The prefrontal cortex also has a key role in suppressing irrelevant stimuli through a mechanism that is not well understood. Recent findings indicate that prefrontal pathways interface with laminar-specific neurochemical classes of inhibitory neurons in sensory cortices, terminate extensively in the frontal and sensory sectors of the inhibitory thalamic reticular nucleus, and target the inhibitory intercalated masses of the amygdala. Circuit-based models suggest that prefrontal pathways can select relevant signals and efficiently suppress distractors, in processes that are disrupted in schizophrenia and in other disorders affecting prefrontal cortices.
\end{abstract}

\section{Keywords}

inhibitory systems; laminar connections; cortical calbindin inhibitory neurons; cortical parvalbumin inhibitory neurons; thalamic reticular nucleus; amygdala; primates

\section{Overview}

Purposive behavior requires selection of relevant information for a specific task and ignoring extraneous stimuli. One can read a book and ignore the humming of the lights, the rain falling against the window, and the myriad stimuli from the background scene impinging on the senses even in a quiet environment. One can also manage to carry out tasks even under challenging conditions, such as having a conversation in a noisy restaurant. There is evidence that the prefrontal cortex in primates is necessary not only for choosing relevant information for the task at hand, but also for actively suppressing irrelevant stimuli. Patients with damage to lateral prefrontal cortex after a stroke, or in aging affecting function of the prefrontal cortex, can perform auditory discrimination tasks, but their performance deteriorates when irrelevant auditory stimuli are introduced (Woods and Knight 1986). In these patients, neural activity in dorsolateral prefrontal areas decreases and activity in auditory cortices increases (Chao and Knight 1998), suggesting the lifting of inhibitory control by prefrontal cortex. Patients with other disorders thought to affect function of lateral prefrontal cortex, such as schizophrenia, or attention deficit hyperactivity disorder, are also readily distracted by extraneous stimuli (Arnsten and Li 2005; Cohen and others 1996).

How does the prefrontal cortex select relevant stimuli and ignore irrelevant stimuli to allow purposive behavior? The mechanism is not well understood, but we're beginning to understand 
the circuits that may underlie this process. The ability to zero in and select what is relevant for a given task likely depends on the extensive communication network that links the prefrontal cortex with other areas that process information arriving from the senses, or gained through experience and stored in memory. In addition, actions depend on motivational factors embedded in an emotional context, and the prefrontal cortex is intricately linked with structures associated with emotions.

The prefrontal cortex is connected in a topographically specific manner with most other cortices, including sensory and high-order association areas, and subcortical structures associated with cognition, memory and emotions (Barbas 1995; Fuster 1989; Goldman-Rakic 1988; Petrides 2000). Moreover, there are differences in the set of connections of lateral prefrontal cortices, medial prefrontal cortices, and orbitofrontal cortices, consistent with their functional specialization [reviewed in (Barbas 2000)]. Briefly, compared to medial or orbitofrontal areas, lateral prefrontal cortices have connections with earlier-processing sensory association cortices and have a key role in keeping information temporarily in mind to accomplish a task at hand. Medial prefrontal cortices in the anterior cingulate have robust connections with medial temporal and hippocampal structures associated with long term memory, and with central autonomic structures associated with expression of emotions. Posterior orbitofrontal cortices have strong and highly specialized connections with the amygdala, and are the most multimodal through connections with high-order sensory association cortices representing each modality. The connections of posterior orbitofrontal cortex are consistent with its functional specialization in evaluating the emotional significance of stimuli. Selection of relevant stimuli for a task is likely accomplished through these robust and specialized connections of the distinct sectors of the prefrontal cortex with other structures.

Considerably less is known about the process of inhibition by the prefrontal cortex. One possible way that prefrontal pathways can actively suppress irrelevant stimuli is through synapsing with at least some inhibitory neurons in other areas, which then inhibit neighboring neurons. Or, prefrontal pathways may target inhibitory systems, allowing disinhibition of other systems and triggering diverse outcomes.

This review focuses on circuits established by prefrontal cortices with excitatory and inhibitory systems using three structures that exemplify the role of the prefrontal cortex in guiding behavior by selecting relevant signals and suppressing distractors. The first is the connection of prefrontal cortices with temporal auditory association cortices, which is an excellent model of prefrontal inhibitory control of other cortices. The second is the projection of the prefrontal cortex to the inhibitory thalamic reticular nucleus, which appears to gate information between the thalamus and the cortex. The third is the specialized connection of posterior orbitofrontal cortex with the amygdala, which has a key role in emotional processes.

\section{Excitatory prefrontal pathways}

In primates, the pathways that link a given cortical area with other cortical or subcortical structures are excitatory glutamatergic pathways (White and Keller 1989). No significant longdistance inhibitory projections have been reported in primates, a situation that differs from rats (Carr and Sesack 2000). These excitatory prefrontal pathways may underlie the selection of relevant information, such as focusing on the traffic lights at an intersection and taking appropriate action.

There is a great deal of information on the responses of neurons to a stimulus, or during specific phases of a behavioral task (Funahashi and Kubota 1994; Fuster 1989; Goldman-Rakic 1988). But choosing the right stimuli for a task is not enough, as it appears to be necessary to actively suppress irrelevant signals, as a driver ignores the detailed features of passing cars, or the contents of the corner flower stand. Since projections across areas are excitatory, the 
prefrontal cortex may exercise inhibitory control by synapsing with at least some inhibitory neurons in other areas. As described below, the prefrontal cortex interfaces with several such systems.

\section{GABAergic inhibition in the cortex}

Inhibition in the cortex is effected through local GABAergic neurons, which synapse with neighboring excitatory pyramidal neurons or other inhibitory neurons and, acting through ionotropic or metabotropic receptors, reduce the excitability of postsynaptic neurons. There are other forms of modulation mediated through non-GABAergic first messenger systems in neural structures (Greengard 2001), including the cortex. Here we focus on GABAergic inhibition because it has a key role in the cortex.

It is estimated that about 25-30 per cent of all neurons in the primate cortex are GABAergic (Jones 1993), though their density varies somewhat across areas. In the prefrontal cortex they make up 25-35 per cent of all neurons (Dombrowski and others 2001). Cortical GABAergic neurons are diverse in phenotype, site of their postsynaptic targets, and in efficacy of inhibitory control (Kawaguchi and Kubota 1997; Somogyi and others 1998; Thomson and Deuchars 1997; White and Keller 1989). Understanding the organization of inhibitory neurons in the cortex is prerequisite to understanding the consequences after stimulation of prefrontal pathways.

A useful way of identifying inhibitory neurons in the cortex of primates is by their expression of the calcium binding proteins parvalbumin, calbindin and calretinin, which comprise largely non-overlapping classes of cortical inhibitory neurons (DeFelipe 1997; Hendry and others 1989). Each of the tree neurochemical classes of inhibitory neurons includes several morphological types. The neurochemical classes can be differentiated by their pattern of distribution in the cortex and mode of inhibition. Calretinin neurons, are found mostly in the upper cortical layers (II-IIIa), and there's evidence that they innervate other GABAergic neurons (DeFelipe and others 1999; Gonchar and Burkhalter 1999; Melchitzky and others 2005; Meskenaite 1997). Parvalbumin and calbindin inhibitory neurons appear to be complementary in density and laminar distribution, at least in prefrontal, superior temporal and parietal cortices, where they have been studied quantitatively (Barbas and others 2005; Dombrowski and others 2001; Medalla and Barbas 2006). In prefrontal limbic areas, for example, there's a much higher density of calbindin than parvalbumin inhibitory neurons, but the density gradually changes through successively neighboring prefrontal cortices, and is about equal in the eulaminate prefrontal areas on the lateral surface (Dombrowski and others 2001). The overall density of inhibitory neurons is comparable across areas, but the relative density of each neurochemical class may differ among cortices.

The parvalbumin and calbindin classes of inhibitory neurons also have complementary laminar distributions, consistent in all cerebral cortices examined [reviewed in (Hof and others 1999)], and distinct modes of inhibiting other neurons (Fig. 1). Parvalbumin neurons are found preferentially in the middle-deep layers of the cortex, and include the morphological types of basket and chandelier inhibitory neurons. Parvalbumin neurons synapse on cell bodies, proximal dendrites and axon initial segments of pyramidal neurons (Kawaguchi and Kubota 1997; Somogyi and others 1998), suggesting robust inhibitory control. Calbindin neurons, on the other hand, are most densely distributed in cortical layers II and III, and include double bouquet neurons that innervate distal dendrites and spines of other neurons (DeFelipe and others 1989; Peters and Sethares 1997; Zaitsev and others 2005), suggesting a modulatory role. 


\section{Rules of laminar patterns of corticocortical connections and their relationship to excitatory and inhibitory neurons}

The distinct laminar distribution of the neurochemical classes of inhibitory neurons has significant implications for cortical processing, because the connections of prefrontal cortices with other cortices, the thalamus, the amygdala, and other subcortical structures also have specific laminar distributions (Fig. 2). Corticocortical connections, in general, vary widely in their laminar distribution, as seen after injection of neural tracers in any cortical area to label its afferent and efferent connections. A given cortical area receives projections from many other areas, and the laminar origin of projection neurons varies among the projection sites. Projections may arise overwhelmingly from the upper layers (II-III) of one area, mostly from the deep layers (V-VI) of another area, or in different laminar proportions in other areas. The axons of the various projection areas also terminate in different layers of the cortex of destination. Some areas innervate the superficial layers (I-upper layer III), others the middledeep layers (deep III to VI), and others all layers (I-VI).

The laminar patterns of corticocortical connections are complex but they appear to be governed by a simple rule based on the structural relationship of pairs of linked areas, which we call the structural model for connections. Structure in this context refers to the number of cortical layers present in a given area, or the overall neuronal density, and can be described quantitatively. The structural model is based on evidence that within any large cortical region, such as the prefrontal, the individual areas vary in structure in a systematic way. In the prefrontal region, the posterior orbitofrontal and anterior cingulate areas, which are limbic, have fewer than six layers and lower cell density than the eulaminate areas on the lateral surface (Fig. 3A-C). Areas adjacent to the limbic have a neuronal density between the above extremes, as shown in the cartoons (Fig. 3D-G).

The difference in structure between linked areas $(\Delta)$ underlies the pattern of their interconnections. Projection neurons originate mostly from the deep layers of areas with fewer layers, or lower neuronal density, and terminate mostly in the upper layers of areas with more layers or higher cell density (Barbas 1986; Barbas and Rempel-Clower 1997; Rempel-Clower and Barbas 2000), such as the connection from $\mathrm{D}^{\prime}$ to $\mathrm{G}^{\prime}$ (Fig. 3). In the reverse direction, projection neurons originate mostly from the upper layers (II-III) of areas with more layers or higher cell density, and their axons terminate mostly in the middle-deep layers of areas with fewer layers or lower neuronal density (Fig. 3, $\mathrm{G}^{\prime}$ to $\mathrm{D}^{\prime}$ ). When two areas have comparable structure, projection neurons originate in both upper (II-III) and deep (V-VI) layers, and their axons terminate in a columnar pattern including all layers (I-VI) (not shown). The distribution of connections among layers thus reflects the relative difference in structure of the linked areas $(\Delta)$, which can be large (e.g., $D^{\prime}$ to $G^{\prime}$ ) or small (e.g., $E^{\prime}$ to $F^{\prime}$ ), so the structural model for connections is relational. In the prefrontal to intraparietal connections the density of neurons in linked pairs of cortices alone is a good predictor of the laminar distribution of connections, even though the differences in neuronal density between areas are small (Medalla and Barbas 2006).

\section{Functional attributes of laminar-specific corticocortical connections}

Projections that terminate in the superficial layers have been referred to as feedback and those that terminate in the middle layers as feedforward (Felleman and Van Essen 1991). Projections originating or terminating in specific layers encounter different microenvironments with respect to functionally distinct local inhibitory neurons, which also show preferential laminar distribution. Feedback projections that terminate in the upper layers of the cortex encounter a microenvironment dominated by calbindin and calretinin inhibitory neurons (Barbas and others 2005; Medalla and Barbas 2006), which are most prevalent in layers II-III of prefrontal and 
other cortices (DeFelipe and others 1999; Dombrowski and others 2001; Glezer and others 1998; Hendry and others 1989) (Fig. 2).

Feedback projections are thought to influence task related neuronal activity (Bar 2003;Lamme and others 1998;Raizada and Grossberg 2003;Ullman 1995). Physiological studies in prefrontal cortex of behaving non-human primates, and theoretical analysis of the neuronal responses, suggest that calbindin inhibitory neurons have a role in enhancing activity of neurons responding to relevant features in a working memory task and suppressing distractors (Wang and others 2004). Feedback projections, in all systems, including corticocortical or thalamocortical, terminate in long horizontal ribbons in cortical layer I, and come in contact with the apical dendrites of pyramidal neurons from other layers. This circuit architecture is ideal for eliminating distractors through interaction with calbindin neurons in adjacent layer II. The predominant terminations of prefrontal pathways in layers I-II of auditory association cortices, which are enriched with calbindin neurons, may have an important role in masking distractors in auditory discrimination tasks (Barbas and others 2005). This circuitry may help explain why patients with damage to dorsolateral prefrontal cortex do not perform well in discrimination tasks when burdened with distracting stimuli.

Feedforward projections, on the other hand, terminate in the middle-deep cortical layers, where they encounter a microenvironment enriched with parvalbumin inhibitory neurons (Fig. 2B, C). In the sensory systems, feedforward pathways relate signals about the features of stimuli from earlier to later processing sensory cortices. The laminar overlap of feedforward projections with parvalbumin inhibitory neurons, which have wide horizontal arbors, may help restrict the signals transmitted from sensory association areas to the prefrontal cortex through lateral inhibition. Physiological studies have shown that stimulation of feedforward cortical pathways leads to monosynaptic excitation followed by disynaptic inhibition at the site of termination (Shao and Burkhalter 1999).

Prefrontal pathways, therefore, interact extensively with excitatory and inhibitory neurons at the site of termination, as seen in temporal cortices (Barbas and others 2005; Germuska and others 2006). At the synaptic level the prefrontal to temporal pathways target for the most part dendritic spines of pyramidal neurons, which are excitatory (Peters and others 1991; White and Keller 1989). In addition, a smaller but significant number of prefrontal axonal boutons synapse with dendritic shafts of inhibitory neurons in superior temporal cortices, both in the upper layers as well as in the middle layers (Germuska and others 2006).

\section{Presynaptic specificity in corticocortical pathways}

An intriguing and consistent feature of prefrontal pathways at the synaptic level is the difference in the size of axonal boutons that terminate in different cortical layers (Fig. 4). In the projections from prefrontal cortices to temporal auditory cortices, boutons terminating in the middle layers are significantly larger than boutons terminating in layer I (Germuska and others 2006). The laminar-specific presynaptic differences in prefrontal axonal boutons are independent of the specific area of origin in prefrontal cortex, or area of destination in superior temporal cortex. For example, figure 4 shows that the differences in bouton size are consistent whether pathways originate in prefrontal area 10 or area 32 and terminate in the same temporal area, or whether they originate in the same prefrontal area and terminate in two different temporal areas. These findings suggest that the differences in the size of terminals by layer may be general in the cortex.

The significance of terminal size is based on evidence that the number of synaptic vesicles is proportional to bouton size in several cortical systems (Germuska and others 2006; Gonchar and Burkhalter 1999). Moreover, the number of synaptic vesicles has been associated with the probability of multivesicular release upon stimulation (Murthy and others 1997). This evidence 
suggests that synaptic efficacy varies in laminar-specific pathways. Large boutons have more mitochondria, which are found in highly active networks. It is possible that large boutons represent facilitated pathways with efficient transmission of signals. In fact, some small boutons do not have mitochondria (Germuska and others 2006).

\section{Relationship of the origin of corticocortical projections to inhibitory neurons}

Corticocortical projection neurons from different layers also appear to be subject to distinct types of inhibition at the site of origin (Fig. 5). Corticocortical projections originating in the lower part of layer III, and in layer $\mathrm{V}$, are likely under the predominant influence of parvalbumin inhibitory neurons, which are prevalent in the middle cortical layers. In the prefrontal to temporal pathway, for example, projection neurons in cingulate area 32 originate from the deep layers (V-VI) (Barbas and others 1999). In contrast, a strong temporally directed projection from prefrontal area 10 arises mostly from the upper layers (II-III). Projections from the cingulate cortex, therefore, emerge from a microenvironment dominated by parvalbumin inhibitory neurons, whereas projection neurons from area 10 are intermingled mostly with calbindin and calretinin inhibitory neurons.

Interestingly, areas 10 and 32 have distinct functional attributes. Area 10 has a key role in complex working memory tasks that require juggling of multiple tasks (Koechlin and others 1999), such as interrupting reading a book to answer the phone, and then remembering to resume reading at the place of departure. This type of working memory would require temporary modulation of neurons that hold in memory the spot where the reader left off, an effect that may be due to the modulatory influence of calbindin inhibitory neurons, which innervate the distal dendrites of pyramidal neurons. Strong inhibition in such a case might lead to forgetting.

On the other hand, the cingulate cortex is engaged when an error is made, or in the presence of conflict. The anterior cingulate is situated in front of cingulate motor areas (Dum and Strick 2002), and its neurons respond when a signal is given to reverse a decision for a specific act (Schall and others 2002). Reversing a command or correcting an error may require strong inhibition of the neurons involved, which may be achieved by local parvalbumin inhibitory neurons, which innervate pyramidal neurons on, or near, the cell body. Inhibition by parvalbumin neurons may prevent transmission of a signal. The differences in the association of area 10 and the cingulate pathway with functionally distinct neurochemical classes of inhibitory neurons may be key to their functional specialization. A future challenge is to model and investigate the consequences of stimulation of specific pathways, based on the laminar regularity of corticocortical connections predicted by the structural model, the density of connections obtained from empirical studies, and the laminar-specific distribution of distinct neurochemical classes of inhibitory neurons.

\section{Projections from cortical areas to the thalamic reticular nucleus}

Pathways from the prefrontal cortex also target to a significant extent subcortical inhibitory systems. The pathway known best in this context is the prefrontal projection to the neostriatal (caudate and putamen) segments of the basal ganglia. The prefrontal cortex is not unique in projecting to the neostriatum, since all other cortices project to the caudate or putamen as well. However, the prefrontal cortex receives privileged information from the striatum through the thalamic mediodorsal and ventral anterior nuclei, a pattern of connections seen also for motor and premotor areas but not other cortices (Haber 2003; Xiao and Barbas 2004).

Another inhibitory subcortical system targeted by the cortex is the thalamic reticular nucleus. The reticular nucleus is composed of a thin mesh of inhibitory neurons and enveils the dorsal thalamus covering its dorsal and lateral aspects and flaring open inferiorly. The reticular 
nucleus is situated between the thalamus and the cortex and has bidirectional connections with the dorsal thalamus, is innervated by projections from the cortex, but does not project to the cortex (Fig. 6). This circuitry suggests that the reticular nucleus may filter information between the thalamus and the cortex and either allow signals to be processed or pass directly to the cortex. This filtering of signals can occur through a process whereby reticular neurons innervate and inhibit thalamic relay neurons or disinhibit them by innervating thalamic inhibitory neurons found in the dorsal thalamus of primates (Jones 1985).

The projection of cortical areas to the reticular nucleus is topographic, and in studies conducted mostly in rodents, it's evident that there's, from back to front of the nucleus, an auditory sector, a visual sector, a somatosensory/visceral sector, and a frontal sector. In addition, the dorsal thalamic nuclei send an axonal branch to the reticular nucleus along their projection to the cortex. The organization of the system is such that cortical areas and the specific thalamic nuclei they are connected with map onto the same parts of the reticular nucleus. There is, therefore, a fair topographic map of the thalamus and the cortex on the reticular nucleus [reviewed in (Guillery and Harting 2003; Pinault 2004)].

\section{The unique features of prefrontal pathways to the thalamic reticular nucleus}

Like other cortices, the prefrontal cortex projects to the reticular nucleus. Axons from prefrontal cortices terminate mostly in the anterior reticular sector, where they overlap with projections from the adjacent motor areas. However, unlike other cortical areas, pathways from several prefrontal areas terminate in the anterior as well as the central and posterior parts of the reticular nucleus, where they overlap with projections from sensory association areas (Zikopoulos and Barbas 2006). This topography suggests that axons from prefrontal cortices may be in a position to influence signals passing from sensory-related thalamic nuclei to the cortex (Fig. 7).

Interestingly, not all prefrontal cortices behave the same way in their projection to the reticular nucleus. Projections from prefrontal areas 10 and 32, for example, remain largely confined to the anterior sector of the reticular nucleus, which also includes the majority of terminations from other prefrontal cortices. This pattern is particularly interesting, because area 32 is a cingulate limbic area, known for its widespread connections with other cortices and with subcortical structures [reviewed in (Barbas and others 2002)]. On the other hand, area 46, which has comparatively more restricted cortical, thalamic, and other subcortical connections, has widespread projections to the reticular nucleus, extending to some of the sensory sectors. Area 46 has a key role in working memory, and its widespread projections to the reticular nucleus may allow selection of sensory and other signals and suppress distractors at an early stage of processing.

\section{The mediodorsal thalamic nucleus has widespread connections with the reticular nucleus}

The mediodorsal nucleus is the chief thalamic nucleus for the prefrontal cortex, although several other thalamic nuclei, including the ventral anterior, intralaminar, midline, the medial pulvinar, and suprageniculate, project to prefrontal cortices as well [reviewed in (Barbas 2000)]. Some thalamic nuclei, including the anterior medial and the midline nuclei project preferentially to the limbic parts of the prefrontal cortex, in the posterior orbitofrontal cortex and anterior cingulate (Dermon and Barbas 1994). From the nuclei that project to at least some prefrontal cortices, the anterior medial and ventral anterior map onto the anterior parts of the reticular nucleus, which are also innervated by prefrontal cortices. On the other hand, the mediodorsal nucleus, which provides more than 80 per cent of the projection neurons from the thalamus directed to lateral prefrontal cortices, including area 46 (Barbas and others 1991), has just as widespread connections with the thalamic reticular nucleus, as area 46 (Fig. 7). Thus, the connections of the mediodorsal nucleus span the anterior three quarters of the thalamic reticular nucleus, at sites that also receive projections from area 46 (Zikopoulos and 
Barbas 2006). This circuitry provides yet another way through which prefrontal areas with executive functions can influence the flow of information through the thalamus. Area 46 and the mediodorsal nucleus may exercise control of sensory signals by synapsing onto reticular neurons that innervate sensory association thalamic nuclei found inside the central and posterior sectors of the reticular nucleus. This circuitry suggests that the prefrontal cortex may affect sensory processing at an early stage through the thalamus.

Area 9, situated on the dorsolateral surface of the prefrontal cortex, is another area with widespread projections to the thalamic reticular nucleus, though the overlap with projections from sensory cortices is not as extensive as for area 46. In addition, area 13, an orbitofrontal region with a role in evaluating the significance of stimuli, also has widespread terminations on the reticular nucleus.

\section{Presynaptic specialization of prefrontal projections to the thalamic reticular nucleus}

Prefrontal projections to the reticular nucleus are also distinguished from other cortical projections in their presynaptic specialization (Fig. 8). Unlike projections from sensory or motor cortices, which terminate only as small boutons and are thought to originate from cortical layer VI, projections from prefrontal cortices also include a significant proportion of large boutons ( 10\%), even though most are small (Zikopoulos and Barbas 2006).

Large terminals from the cortex to the thalamus have been associated with 'driver' input (Guillery and Sherman 2002), ensuring the passage of a signal to the next brain station. Prefrontal cortices thus differ from other cortices by issuing a significant proportion of driverlike projections to the thalamic reticular nucleus. As noted earlier for prefrontal to temporal pathways, large boutons have more synaptic vesicles, suggesting increased probability of multivesicular release upon stimulation. Large boutons, therefore, may ensure activation of reticular neurons. At least in rodents, reticular neurons are coupled by electrical synapses (Landisman and others 2002), so that even a small number of large boutons may initiate widespread activation in the nucleus. Interestingly, even though large boutons make up a small proportion of all terminals in the reticular nucleus, they are just as widespread as the small boutons, suggesting that they have a global effect. This feature of the prefrontal terminations may provide a mechanism through which the prefrontal cortex can suppress distracting stimuli at the level of the thalamus.

What is the mechanism through which the prefrontal cortex may select relevant stimuli and suppress distractors through the thalamus? To explore this issue it's necessary to return to the basic circuitry of the reticular nucleus with the cortex and dorsal thalamus, using sensory input as an example. Sensory signals from the periphery are transmitted to sensory relay nuclei of the dorsal thalamus and then to the cortex. As shown in Figure 6, relay neurons from the dorsal thalamus also send an axonal offshoot to the reticular nucleus on their projection to the cortex. The reticular nucleus receives a signal from the relay neuron and communicates back with the dorsal thalamus, engaging one of two distinct circuits identified in anatomic and physiologic studies (Pinault 2004). One circuit is known as an open loop, meaning that a reticular neuron does not project back to the thalamic relay neuron that transmitted the signal, but projects to another relay neuron, or to a thalamic inhibitory neuron. If the reticular neuron innervates an inhibitory neuron that projects to the relay neuron that received the peripheral signal, this would amount to disinhibition of the relay neuron, prolonging its ability to transmit to the cortex (Fig. 6 , dotted axons). The other circuit is a closed loop, where the reticular neuron innervated by a thalamic relay neuron carrying a peripheral signal projects back to the relay neuron and inhibits it (Fig. 6, solid axons). The closed loop suggests that a peripheral input is sent briefly to the cortex before the thalamic relay neuron is inhibited by the reticular neuron. The peripheral input can be salient or distracting, and either stimulus can go through an open or a closed loop. 
Moreover, salient and distracting stimuli impinging on the subject can be processed through combinations of open and closed loops, four combinations in total.

The wiring diagram in Figure 9 summarizes the cortical and thalamic connections of the reticular nucleus. As shown, the processing of sensory stimuli and suppression of distractors can be handled to various degrees of success by the sensory systems (Guillery and others 1998;McAlonan and Brown 2002). Then what is the potential role of the prefrontal projection? The model in Figure 9 shows that the prefrontal cortex can magnify the drive for transmission of salient signals and at the same time efficiently suppress distractors. The prefrontal cortex can achieve these dual functions as suggested by its special anatomic features: widespread projections that include the sensory sectors of the reticular nucleus; large terminals that suggest efficient transfer of information; robust connections with the mediodorsal thalamic nucleus, which also has widespread projections that map onto the same parts of the reticular nucleus as the prefrontal projections. This pattern of innervation by select prefrontal areas increases the probability that salient stimuli are sent to the cortex and distractors are suppressed, whereas the sensory cortices alone may fail. The circuits from prefrontal cortex, the mediodorsal nucleus and the reticular nucleus suggest top-down modulation by prefrontal cortex at an early stage of sensory processing.

\section{Specialization of posterior orbitofrontal connections with the amygdala}

We began by stating that the prefrontal cortex selects relevant information and suppresses irrelevant signals. But choices in everyday life are governed by motives and drives, embedded in an emotional context. The prefrontal cortex has limbic components, situated in the anterior cingulate and caudal orbitofrontal region (Fig. 3A, B), which are connected with other prefrontal cortices and a host of other cortical and subcortical limbic structures associated with emotional processing. Limbic cortices, in general, are situated at the edge of each cortical system on the medial and basal aspects of the cerebral hemispheres, and include the cingulate and retrosplenial cortices, medial temporal, temporal polar, and caudal orbitofrontal cortices. Limbic cortices have widespread connections with other limbic cortices, association cortices, and with subcortical limbic structures, including the amygdala, the hypothalamus, the hippocampus, midline and anterior thalamic nuclei.

Limbic prefrontal areas have the most robust and bidirectional connections with the amygdala. This applies for both the cingulate and posterior orbitofrontal cortex, but the latter shows further specialization in its connections with the amygdala. The posterior orbitofrontal cortex stands apart among prefrontal cortices as the most multimodal, sampling the entire sensory periphery through projections from cortices associated with each sensory modality (Fig. 10). Moreover, as one of the two limbic sectors of the prefrontal cortex, it is connected with both cortical and subcortical limbic structures. The caudal orbitofrontal region has strong two-way connections with the amygdala [reviewed in (Barbas and Zikopoulos 2006)]. Moreover, the connections of the posterior orbitofrontal cortex with the amygdala are the most specialized among prefrontal cortices, and perhaps among all cortices. Unlike other prefrontal areas, the input and output connections of the posterior orbitofrontal cortex in the amygdala are partly segregated in rhesus monkeys (Ghashghaei and Barbas 2002). Most projection neurons from the amygdala directed to orbitofrontal cortex are found in the basolateral, basomedial (also known as accessory basal), and lateral nuclei, and a few are found in the cortical nuclei of the amygdala. Projections from orbitofrontal cortex to the amygdala terminate mostly in the same nuclei. But the densest terminations from posterior orbitofrontal cortex target an entirely inhibitory system in the amygdala, the intercalated masses (Ghashghaei and Barbas 2002). These small GABAergic neurons form small clusters between different nuclei of the amygdala, as their name implies (Moga and Gray 1985;Paré and Smith 1993;Pitkanen and Amaral 1993). Like the reticular nucleus of the thalamus, the intercalated masses do not project to the cortex, but have significant 
bidirectional connections with other nuclei of the amygdala. The unique circuitry between the posterior orbitofrontal cortex and the amygdala suggests a special relationship between the two structures in the processing of emotional information.

The targeting by posterior orbitofrontal cortex of a specialized inhibitory system in the amygdala has significant implications for the internal processing of information in the amygdala, as summarized in Figure 10. The intercalated masses project prominently to the central nucleus of the amygdala and inhibit it (Paré and Smith 1993). In a circuit of sequential inhibitory systems, reminiscent of the projections from the cortex to the basal ganglia, the central nucleus sends inhibitory projections to the hypothalamus and the brainstem, targeting central autonomic structures. When the orbitofrontal pathway is activated, the circuitry suggests disinhibition of central autonomic structures, which then can be activated through projections from other structures. In turn, central autonomic structures project to spinal autonomic nuclei that innervate peripheral autonomic organs, such as the heart and the lungs. Activation of the pathway from the orbitofrontal cortex to the intercalated masses, therefore, could lead to a cascade of events resulting in increased autonomic drive and increased activity of peripheral autonomic organs, as seen during emotional arousal. Central autonomic structures may be activated through direct projections from limbic cortices. Anterior cingulate areas, in particular, synapse with hypothalamic structures through large endings, suggesting efficient activation (Barbas and others 2003).

Another specialized pathway from the posterior orbitofrontal cortex terminates in the central nucleus of the amygdala, is also unidirectional, but lighter than the pathway to the intercalated masses (Carmichael and Price 1995; Ghashghaei and Barbas 2002). As noted above, the central amygdalar nucleus issues inhibitory projections to central autonomic structures. Consequently, activation of the orbitofrontal pathway to the central nucleus would result in inhibition of the hypothalamus and brainstem, preventing activation of spinal autonomic structures. The direct projection from orbitofrontal cortex to the central nucleus of the amygdala suggests a mechanism through which heightened autonomic activity may be reduced and the system can then return to autonomic homeostasis. This pathway may be activated when an emotionally charged situation changes and a person calms down.

The ascending pathways from the amygdala to prefrontal cortex are equally complex, innervating robustly in a columnar pattern several sites of posterior orbitofrontal and anterior cingulate cortices. But axons from the amygdala reach all prefrontal cortices, albeit to a different extent, targeting heavily the superficial layers (Ghashghaei and others 2006). In layer II of prefrontal cortex dense terminations from the amygdala are intermingled with calbindin inhibitory neurons. Based on the proposed role of calbindin neurons in enhancing relevant signals and suppressing distractors, we can speculate that they may help focus attention on motivationally relevant stimuli.

The above discussion indicates that the medial, orbitofrontal, and lateral sectors of the prefrontal cortex are specialized in their connections and contribute to specific aspects of a task. But the three prefrontal sectors are also linked through sequential corticocortical pathways, allowing the integration of information arriving from the senses, memory and the internal emotional environment. The prefrontal cortex can guide behavior through projections that engage mechanisms of excitation and inhibition in other cortices and subcortical structures. Pathology of lateral prefrontal cortices, as seen in schizophrenia, disrupts the ability to focus on relevant stimuli and ignore distractors. These deficits may reflect reduced influence of prefrontal pathways on inhibitory cortical systems, diminished filtering of signals through the dorsal thalamus and the reticular nucleus, and reduction of the influence of the thalamic mediodorsal nucleus. Interestingly, the posterior orbitofrontal cortex, which is associated with emotions, also maps widely onto TRN. Pathology of the orbitofrontal cortex likely affects the 
specialized pathways through the amygdala that allow focusing on motivationally relevant stimuli, increase autonomic drive in emotional arousal, and return to homeostasis, as the circumstances demand. Diseases marked by anxiety may reflect abnormally heightened activation of the pathway from posterior orbitofrontal cortex to the thalamus and the intercalated masses of the amygdala.

\section{Acknowledgments}

The authors' work was supported by NIH grants from NINDS and NIMH.

\section{References}

Arnsten AF, Li BM. Neurobiology of executive functions: catecholamine influences on prefrontal cortical functions. Biol Psychiatry 2005;57:1377-1384. [PubMed: 15950011]

Bar M. A cortical mechanism for triggering top-down facilitation in visual object recognition. J Cogn Neurosci 2003;15:600-609. [PubMed: 12803970]

Barbas H. Pattern in the laminar origin of corticocortical connections. J Comp Neurol 1986;252:415422. [PubMed: 3793985]

Barbas H. Anatomic basis of cognitive-emotional interactions in the primate prefrontal cortex. Neurosci Biobehav Rev 1995;19:499-510. [PubMed: 7566750]

Barbas H. Complementary role of prefrontal cortical regions in cognition, memory and emotion in primates. Adv Neurol 2000;84:87-110. [PubMed: 11091860]

Barbas H, Ghashghaei H, Dombrowski SM, Rempel-Clower NL. Medial prefrontal cortices are unified by common connections with superior temporal cortices and distinguished by input from memoryrelated areas in the rhesus monkey. J Comp Neurol 1999;410:343-367. [PubMed: 10404405]

Barbas, H.; Ghashghaei, H.; Rempel-Clower, N.; Xiao, D. Anatomic basis of functional specialization in prefrontal cortices in primates In: Grafman J, editor Handbook of Neuropsychology. Amsterdam: Elsevier Science; 2002. p. 1-27.

Barbas H, Henion TH, Dermon CR. Diverse thalamic projections to the prefrontal cortex in the rhesus monkey. J Comp Neurol 1991;313:65-94. [PubMed: 1761756]

Barbas H, Medalla M, Alade O, Suski J, Zikopoulos B, Lera P. Relationship of prefrontal connections to inhibitory systems in superior temporal areas in the rhesus monkey. Cereb Cortex 2005;15:13561370. [PubMed: 15635060]

Barbas H, Rempel-Clower N. Cortical structure predicts the pattern of corticocortical connections. Cereb Cortex 1997;7:635-646. [PubMed: 9373019]

Barbas H, Saha S, Rempel-Clower N, Ghashghaei T. Serial pathways from primate prefrontal cortex to autonomic areas may influence emotional expression. BMC Neurosci 2003;4:25. [PubMed: 14536022]

Barbas, H.; Zikopoulos, B. Sequential and parallel circuits for emotional processing in primate orbitofrontal cortex. In: Zald, D.; Rauch, S., editors. The Orbitofrontal Cortex. 1st. New York: Oxford University Press; 2006. p. 57-91.

Carmichael ST, Price JL. Limbic connections of the orbital and medial prefrontal cortex in macaque monkeys. J Comp Neurol 1995;363:615-641. [PubMed: 8847421]

Carr DB, Sesack SR. GABA-containing neurons in the rat ventral tegmental area project to the prefrontal cortex. Synapse 2000;38:114-123. [PubMed: 11018785]

Chao LL, Knight RT. Contribution of human prefrontal cortex to delay performance. J Cogn Neurosci 1998;10:167-177. [PubMed: 9555105]

Cohen JD, Braver TS, O'Reilly RC. A computational approach to prefrontal cortex, cognitive control and schizophrenia: recent developments and current challenges. Philos Trans R Soc Lond B Biol Sci 1996;351:1515-1527. [PubMed: 8941963]

DeFelipe J. Types of neurons, synaptic connections and chemical characteristics of cells immunoreactive for calbindin-D28K, parvalbumin and calretinin in the neocortex. J Chem Neuroanat 1997;14:1-19. [PubMed: 9498163] 
DeFelipe J, Gonzalez-Albo MC, del Rio MR, Elston GN. Distribution and patterns of connectivity of interneurons containing calbindin, calretinin, and parvalbumin in visual areas of the occipital and temporal lobes of the macaque monkey. J Comp Neurol 1999;412:515-526. [PubMed: 10441237]

DeFelipe J, Hendry SH, Jones EG. Synapses of double bouquet cells in monkey cerebral cortex visualized by calbindin immunoreactivity. Brain Res 1989;503:49-54. [PubMed: 2611658]

Dermon CR, Barbas H. Contralateral thalamic projections predominantly reach transitional cortices in the rhesus monkey. J Comp Neurol 1994;344:508-531. [PubMed: 7523458]

Dombrowski SM, Hilgetag CC, Barbas H. Quantitative architecture distinguishes prefrontal cortical systems in the rhesus monkey. Cereb Cortex 2001;11:975-988. [PubMed: 11549620]

Dum RP, Strick PL. Motor areas in the frontal lobe of the primate. Physiol Behav 2002;77:677-682. [PubMed: 12527018]

Felleman DJ, Van Essen DC. Distributed hierarchical processing in the primate cerebral cortex. Cereb Cortex 1991;1:1-47. [PubMed: 1822724]

Funahashi S, Kubota K. Working memory and prefrontal cortex. Neurosci Res 1994;21:1-11. [PubMed: 7708289]

Fuster, JM. The Prefrontal Cortex. New York: Raven Press; 1989.

Germuska M, Saha S, Fiala J, Barbas H. Synaptic distinction of laminar specific prefrontal-temporal pathways in primates. Cereb Cortex 2006;16:865-875. [PubMed: 16151179]

Ghashghaei HT, Barbas H. Pathways for emotions: Interactions of prefrontal and anterior temporal pathways in the amygdala of the rhesus monkey. Neuroscience 2002;115:1261-1279. [PubMed: 12453496]

Ghashghaei HT, Hilgetag CC, Barbas H. Sequence of information processing for emotions based on the anatomic dialogue between prefrontal cortex and amygdala. Neuroimage. 2006

Glezer II, Hof PR, Morgane PJ. Comparative analysis of calcium-binding protein-immunoreactive neuronal populations in the auditory and visual systems of the bottlenose dolphin (Tursiops truncatus) and the macaque monkey (Macaca fascicularis). J Chem Neuroanat 1998;15:203-237. [PubMed: 9860088]

Goldman-Rakic PS. Topography of cognition: Parallel distributed networks in primate association cortex. Ann Rev Neurosci 1988;11:137-156. [PubMed: 3284439]

Gonchar Y, Burkhalter A. Connectivity of GABAergic calretinin-immunoreactive neurons in rat primary visual cortex. Cereb Cortex 1999;9:683-696. [PubMed: 10554991]

Greengard P. The neurobiology of slow synaptic transmission. Science 2001;294:1024-1030. [PubMed: 11691979]

Guillery RW, Feig SL, Lozsàdi DA. Paying attention to the thalamic reticular nucleus. Trends Neurosci 1998;21:28-32. [PubMed: 9464683]

Guillery RW, Harting JK. Structure and connections of the thalamic reticular nucleus: Advancing views over half a century. J Comp Neurol 2003;463:360-371. [PubMed: 12836172]

Guillery RW, Sherman SM. Thalamic relay functions and their role in corticocortical communication: generalizations from the visual system. Neuron 2002;33:163-175. [PubMed: 11804565]

Haber SN. The primate basal ganglia: parallel and integrative networks. J Chem Neuroanat 2003;26:317330. [PubMed: 14729134]

Hendry SHC, Jones EG, Emson PC, Lawson DEM, Heizmann CW, Streit P. Two classes of cortical GABA neurons defined by differential calcium binding protein immunoreactivities. Exp Brain Res 1989;76:467-472. [PubMed: 2767197]

Hof PR, Glezer II, Conde F, Flagg RA, Rubin MB, Nimchinsky EA, et al. Cellular distribution of the calcium-binding proteins parvalbumin, calbindin, and calretinin in the neocortex of mammals: phylogenetic and developmental patterns. J Chem Neur 1999;16:77-116.

Jones, EG. The Thalamus. New York: Plenum Press; 1985.

Jones EG. GABAergic neurons and their role in cortical plasticity in primates. Cereb Cortex 1993;3:361372. [PubMed: 8260806]

Kawaguchi Y, Kubota Y. GABAergic cell subtypes and their synaptic connections in rat frontal cortex. Cereb Cortex 1997;7:476-486. [PubMed: 9276173] 
Koechlin E, Basso G, Pietrini P, Panzer S, Grafman J. The role of the anterior prefrontal cortex in human cognition. Nature 1999;399:148-151. [PubMed: 10335843]

Lamme VA, Super H, Spekreijse H. Feedforward, horizontal, and feedback processing in the visual cortex. Curr Opin Neurobiol 1998;8:529-535. [PubMed: 9751656]

Landisman CE, Long MA, Beierlein M, Deans MR, Paul DL, Connors BW. Electrical synapses in the thalamic reticular nucleus. J Neurosci 2002;22:1002-1009. [PubMed: 11826128]

McAlonan K, Brown VJ. The thalamic reticular nucleus: more than a sensory nucleus? Neuroscientist 2002;8:302-305. [PubMed: 12194498]

Medalla M, Barbas H. Diversity of laminar connections linking periarcuate and lateral intraparietal areas depends on cortical structure. Eur J Neurosci 2006;23:161-179. [PubMed: 16420426]

Melchitzky DS, Eggan SM, Lewis DA. Synaptic targets of calretinin-containing axon terminals in macaque monkey prefrontal cortex. Neuroscience 2005;130:185-195. [PubMed: 15561434]

Meskenaite V. Calretinin-immunoreactive local circuit neurons in area 17 of the cynomolgus monkey, Macaca fascicularis. J Comp Neurol 1997;379:113-132. [PubMed: 9057116]

Moga MM, Gray TS. Peptidergic efferents from the intercalated nuclei of the amygdala to the parabrachial nucleus in the rat. Neurosci Lett 1985;61:13-18. [PubMed: 2867498]

Murthy VN, Sejnowski TJ, Stevens CF. Heterogeneous release properties of visualized individual hippocampal synapses. Neuron 1997;18:599-612. [PubMed: 9136769]

Paré D, Smith Y. The intercalated cell masses project to the central and medial nuclei of the amygdala in cats. Neuroscience 1993;57:1077-1090. [PubMed: 8309544]

Peters, A.; Palay, SL.; Webster, HD. The fine structure of the nervous system Neurons and their supporting cells. 3rd. New York: Oxford University Press; 1991.

Peters A, Sethares C. The organization of double bouquet cells in monkey striate cortex. J Neurocytol 1997;26:779-797. [PubMed: 9482155]

Petrides M. The role of the mid-dorsolateral prefrontal cortex in working memory. Exp Brain Res 2000;133:44-54. [PubMed: 10933209]

Pinault D. The thalamic reticular nucleus: structure, function and concept. Brain Res Brain Res Rev 2004;46:1-31. [PubMed: 15297152]

Pitkanen A, Amaral DG. Distribution of calbindin-D28k immunoreactivity in the monkey temporal lobe: the amygdaloid complex. J Comp Neurol 1993;331:199-224. [PubMed: 7685361]

Raizada RD, Grossberg S. Towards a theory of the laminar architecture of cerebral cortex: computational clues from the visual system. Cereb Cortex 2003;13:100-113. [PubMed: 12466221]

Rempel-Clower NL, Barbas H. The laminar pattern of connections between prefrontal and anterior temporal cortices in the rhesus monkey is related to cortical structure and function. Cereb Cortex 2000;10:851-865. [PubMed: 10982746]

Schall JD, Stuphorn V, Brown JW. Monitoring and control of action by the frontal lobes. Neuron 2002;36:309-322. [PubMed: 12383784]

Shao Z, Burkhalter A. Role of GABAB receptor-mediated inhibition in reciprocal interareal pathways of rat visual cortex. J Neurophysiol 1999;81:1014-1024. [PubMed: 10085329]

Somogyi P, Tamas G, Lujan R, Buhl EH. Salient features of synaptic organisation in the cerebral cortex. Brain Res Brain Res Rev 1998;26:113-135. [PubMed: 9651498]

Thomson AM, Deuchars J. Synaptic interactions in neocortical local circuits: dual intracellular recordings in vitro. Cereb Cortex 1997;7:510-522. [PubMed: 9276176]

Ullman S. Sequence seeking and counter streams: a computational model for bidirectional information in the visual cortex. Cereb Cortex 1995;5:1-11. [PubMed: 7719126]

Wang XJ, Tegner J, Constantinidis C, Goldman-Rakic PS. Division of labor among distinct subtypes of inhibitory neurons in a cortical microcircuit of working memory. Proc Natl Acad Sci U S A 2004;101:1368-1373. [PubMed: 14742867]

White, EL.; Keller, A. Cortical circuits. Synaptic organization of the cerebral cortex. Structure, function and theory. Boston, MA: Birkhäuser; 1989.

Woods DL, Knight RT. Electrophysiologic evidence of increased distractibility after dorsolateral prefrontal lesions. Neurology 1986;36:212-216. [PubMed: 3945393] 
Xiao D, Barbas H. Circuits through prefrontal cortex, basal ganglia, and ventral anterior nucleus map pathways beyond motor control. Thalamus \& Related Systems 2004;2:325-343.

Zaitsev A, Gonzalez-Burgos G, Povysheva N, Kroner S, Lewis D, Krimer L. Localization of calciumbinding proteins in physiologically and morphologically characterized interneurons of monkey dorsolateral prefrontal cortex. Cereb Cortex 2005;15:1178-1186. [PubMed: 15590911]

Zikopoulos B, Barbas H. Prefrontal projections to the thalamic reticular nucleus form a unique circuit for attentional mechanisms. J Neurosci 2006;26:7348-7361. [PubMed: 16837581] 


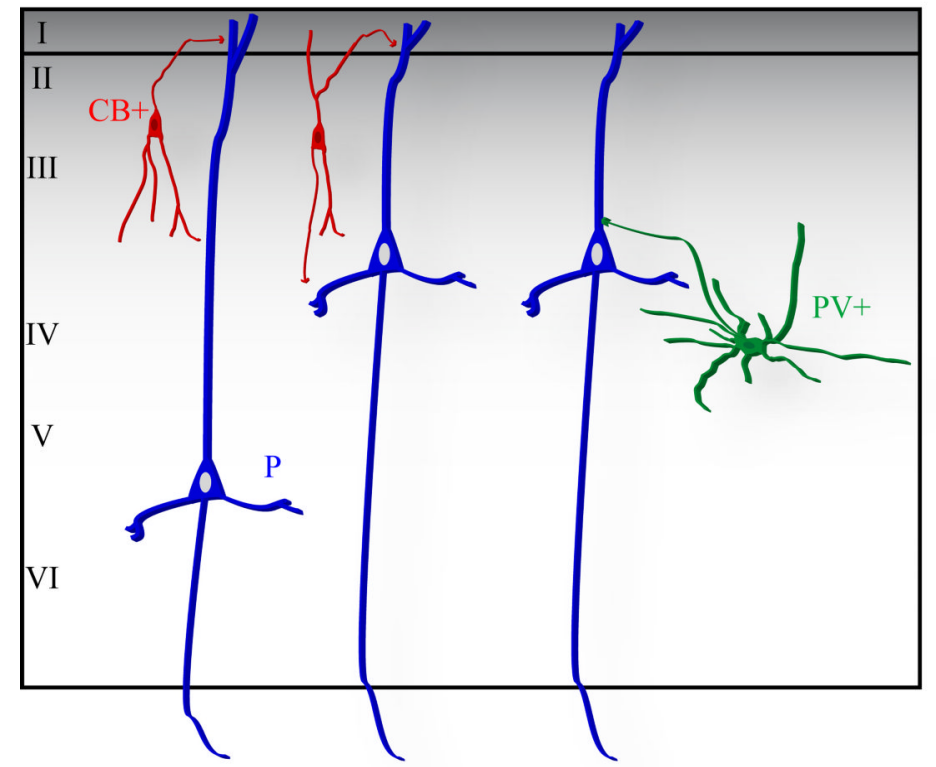

Figure 1.

Laminar distribution and pattern of innervation of two neurochemical classes of inhibitory neurons in the cortex. Calbindin neurons $(\mathrm{CB}, \mathrm{red})$ are preferentially found in the superficial cortical layers and innervate the distal dendrites of pyramidal neurons (blue) from other layers. Parvalbumin inhibitory neurons (PV, green) are preferentially found in the middle layers of the cortex, and innervate the proximal dendrites, soma, and axon initial segment of pyramidal neurons. 

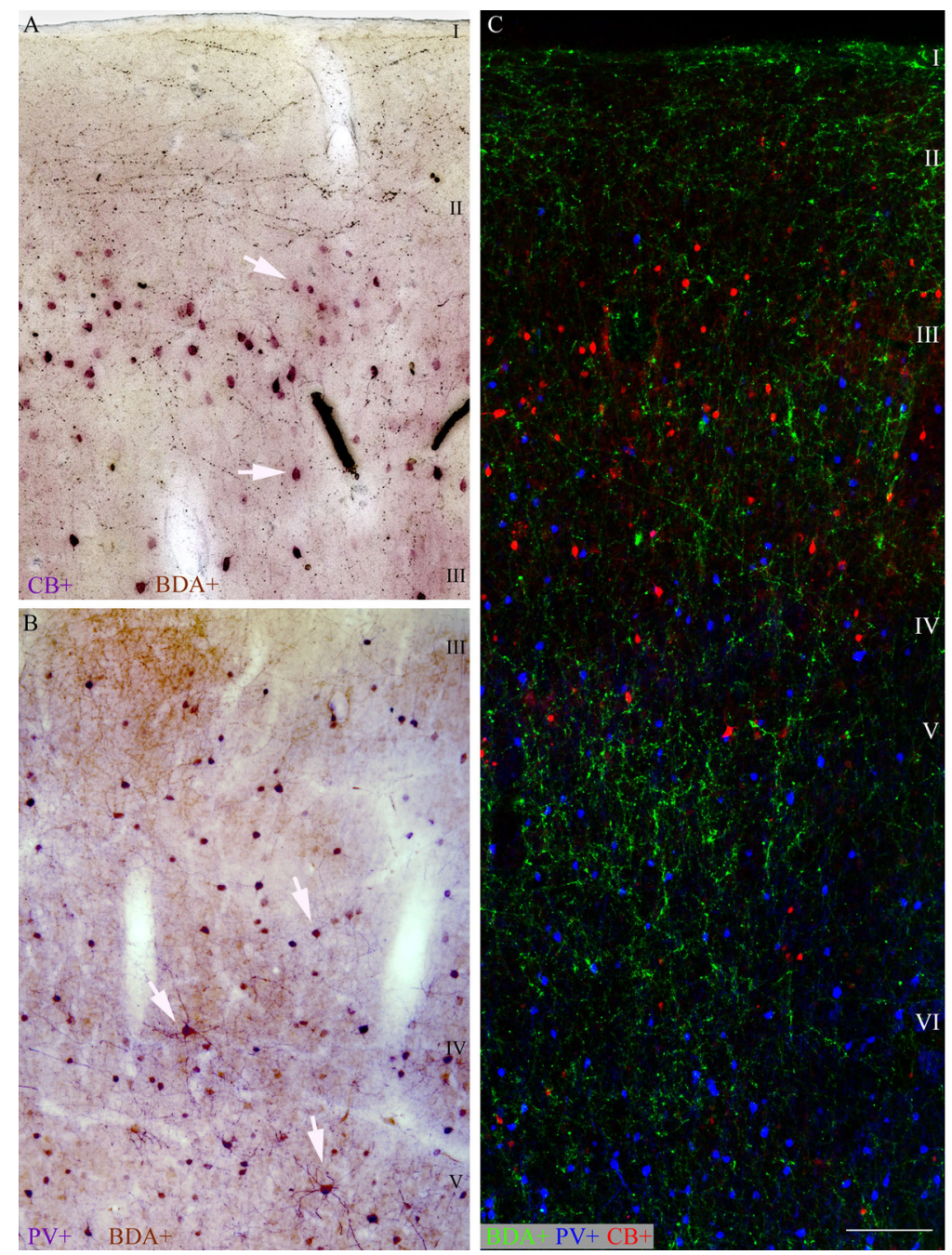

Figure 2.

Relationship of prefrontal pathways to neurochemical classes of inhibitory neurons. $\boldsymbol{A}$, Prefrontal pathways from area 10 terminating in the superficial layers (brown fibers and boutons) of superior temporal area Ts2 are intermingled with CB inhibitory neurons (purple). $\boldsymbol{B}$, Prefrontal pathways from area 32 terminating in the middle cortical layers (brown fibers and boutons) of auditory association area Ts 2 are intermingled with PV inhibitory neurons (purple). $\boldsymbol{C}$, Prefrontal pathways from area 32 (green) terminating in area 9 are intermingled with calbindin inhibitory neurons in the superficial layers (red) and parvalbumin inhibitory neurons (blue) in the middle and deep layers. Labeled pathways in $\mathrm{C}$ were superimposed on matched sections labeled for PV or CB. Scale bar: $100 \mu \mathrm{m}$. 


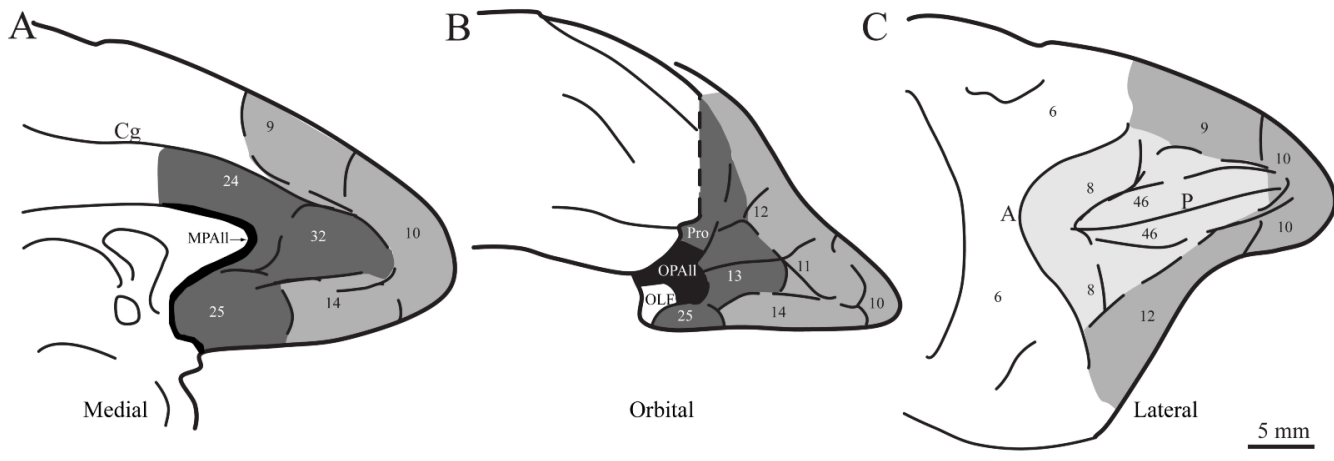

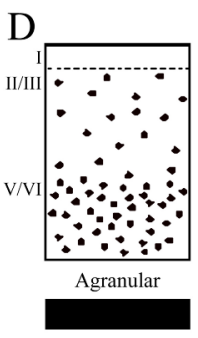

$\mathrm{D}^{\prime}$

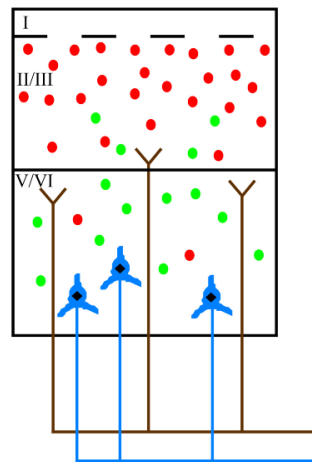

E

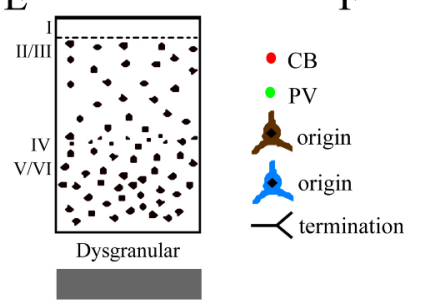

$\mathrm{E}^{\prime}$

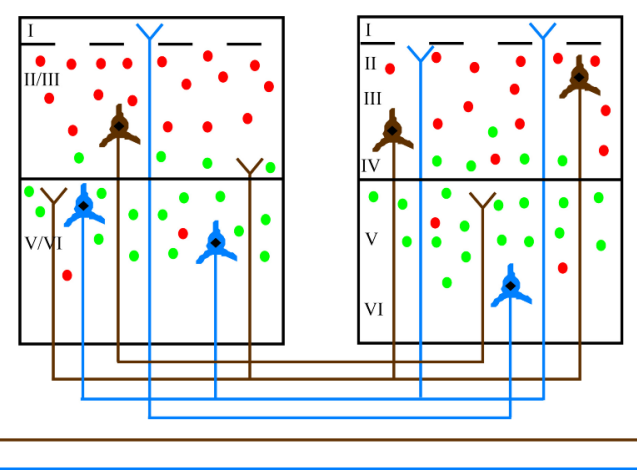

G
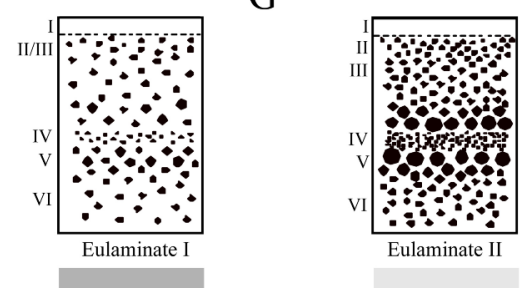

$\mathrm{G}^{\prime}$

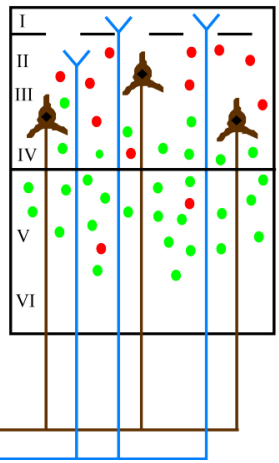

Figure 3.

Cortical structure as the basis of laminar patterns of cortical connections, and their relationship to two neurochemical classes of inhibitory neurons in the cortex. The structural model for connections is depicted for the prefrontal cortex but applies to other cortical regions as well. Top panels: $\boldsymbol{A}-\boldsymbol{C}$, Map of the prefrontal cortex in the rhesus monkey showing the medial, orbitofrontal, and lateral sectors of the prefrontal cortex. Shading shows the various types of cortex. $\boldsymbol{D}-\boldsymbol{G}$, Cortical architecture. The cartoons depict types of cortex showing gradual changes in structure from agranular $(\boldsymbol{D})$, through dysgranular $(\boldsymbol{E})$, eulaminate I $(\boldsymbol{F})$ and eulaminate II $(\boldsymbol{G})$ cortices. Agranular and dysgranular areas have fewer than six layers. The cortical types show progressive change in the density of neurons from low (D) to high (G). $D$ '- $G$ ', The prefrontal cortical types depicted also show a decrease in the density of calbindin (red) and increase in parvalbumin (green) inhibitory neurons from $\mathrm{D}^{\prime}-\mathrm{G}^{\prime}$. Connections between areas with large difference in structure (e.g., from $\mathrm{D}^{\prime}$ to $\mathrm{G}^{\prime}$ ) show a readily distinguishable pattern. Projection neurons originate mostly in the deep layers of areas with fewer layers (e.g., $\mathrm{D}^{\prime}$, blue), or lower neuronal density, and their axons terminate mostly in the upper layers of areas with more layers and higher neuronal density (e.g., $\left.G^{\prime}\right)$. The opposite is seen for the reciprocal connections ( $\mathrm{G}^{\prime}$ to $\mathrm{D}^{\prime}$, brown). $\boldsymbol{E}^{\prime}, \boldsymbol{F}^{\prime}$, A less extreme version of the above pattern is predicted in the interconnections of areas with a moderate difference in structure. The relative laminar distribution and density of CB and PV inhibitory neurons is superimposed on the 
pattern of connections. Layers are depicted in pairs (e.g., II-III) in the agranular and dysgranular areas (collectively called limbic) because the layers cannot be delineated. The structural model was adapted from (Barbas and Rempel-Clower 1997) and the density of CB, PV and neurons in prefrontal cortices from (Dombrowski and others 2001). 

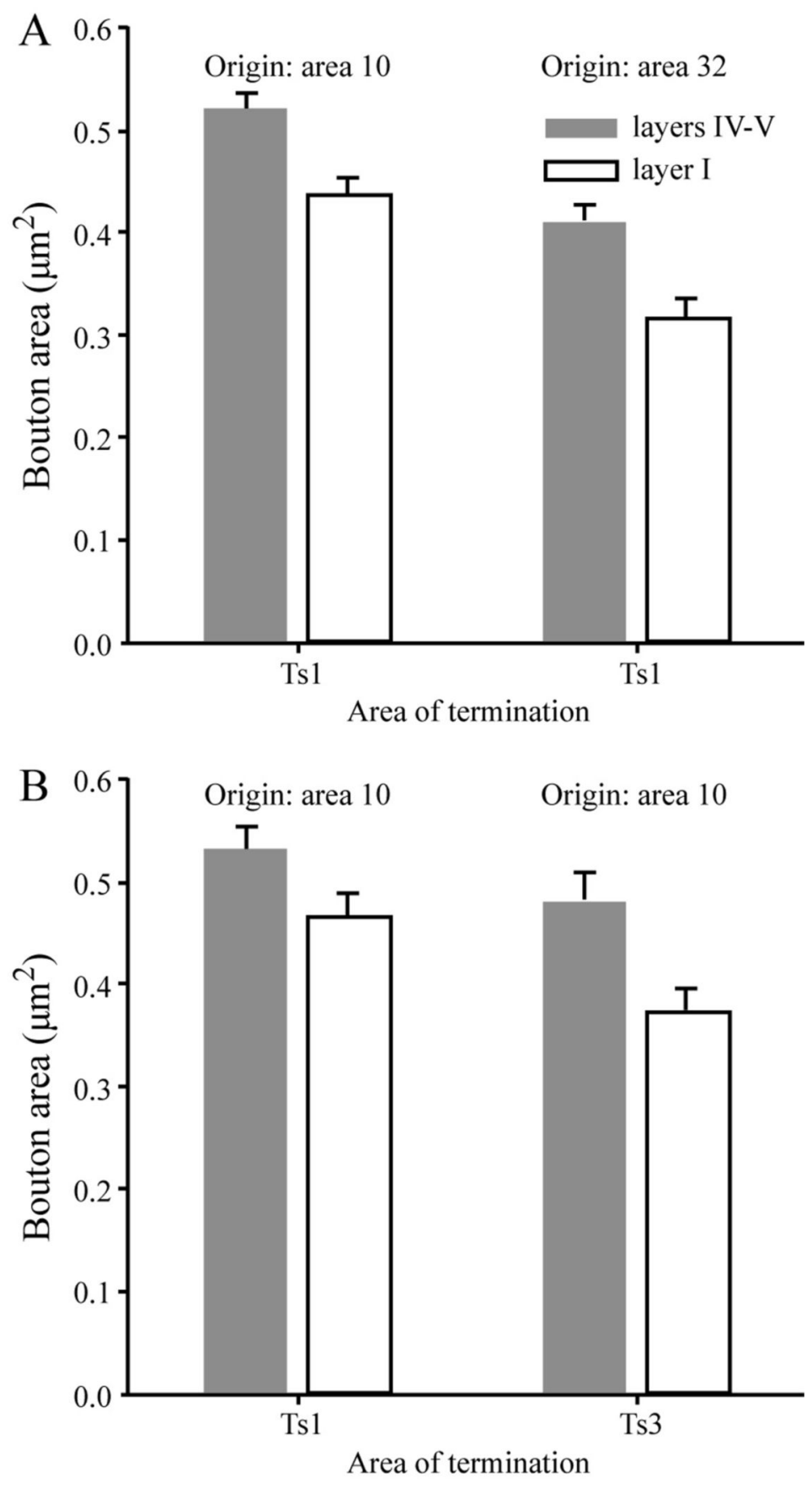

Figure 4.

Prefrontal axonal boutons synapsing in the middle layers are larger than boutons synapsing in layer I of superior temporal auditory association cortex. $\boldsymbol{A}$, Bouton size differences by layer are seen regardless of origin in prefrontal area 10 or cingulate area 32 . $\boldsymbol{B}$, Bouton size differences from axons originating in area 10 are seen regardless of their termination in architectonically distinct superior temporal cortices Ts1 or Ts3. From (Germuska and others 2006). 

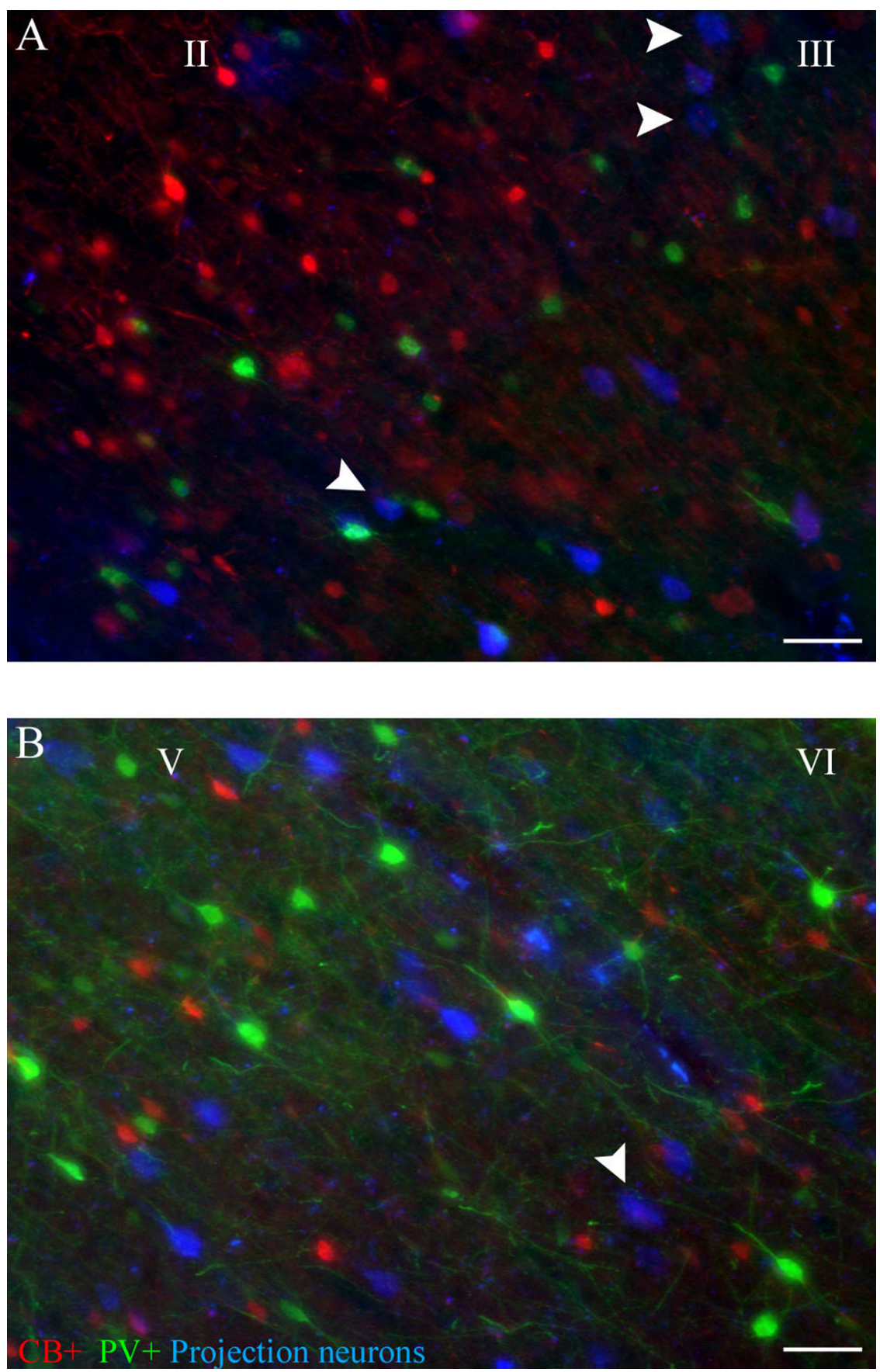

Figure 5.

Relationship of the origin of projection neurons to neurochemical classes of inhibitory neurons. $\boldsymbol{A}$, Projection neurons originating in the superficial layers (blue neurons) of superior temporal cortex are intermingled mostly with local CB inhibitory neurons (red). B, Projection neurons originating in the middle-deep cortical layers (blue neurons) of superior temporal cortex are intermingled mostly with local PV inhibitory neurons (green). The labeled neurons are from temporal auditory area Ts1 and project to prefrontal area 9. Scale bar, $50 \mu \mathrm{m}$. 


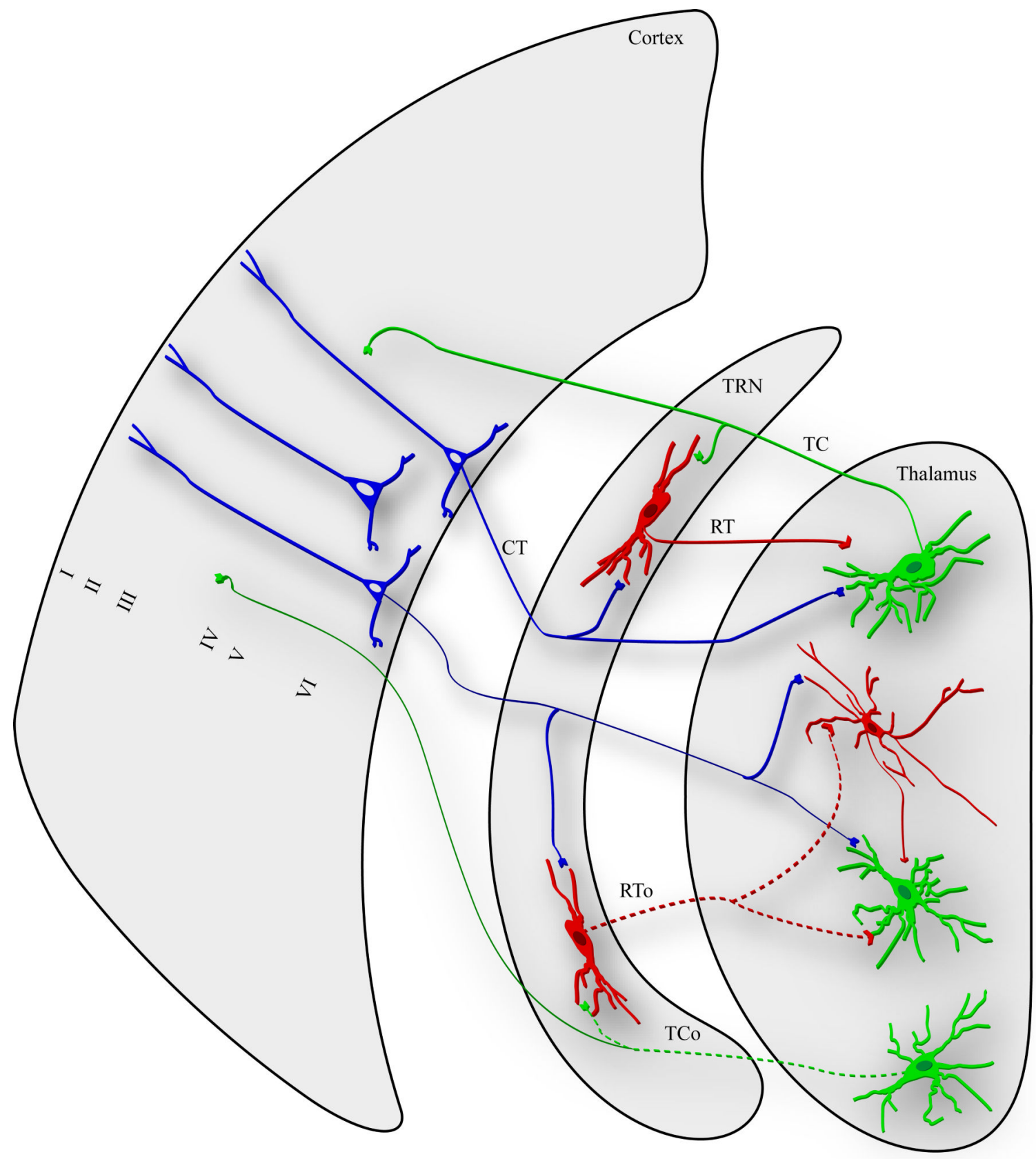

Figure 6.

The position of the thalamic reticular nucleus (TRN) and its innervation by the cortex and thalamus. The TRN has reciprocal connections with the thalamus (right) and receives unidirectional projections from the cortex (left; CT). The connections of TRN with the thalamus are organized either as closed loops (solid processes), or open loops (dotted processes). In the closed loop circuit, a thalamic relay neuron projects to a reticular neuron (top; TC), and the TRN neuron innervates and inhibits the relay neuron (RT). In the open loop circuit, a thalamic relay neuron (green, bottom) projects to a TRN neuron (TCo), which innervates and inhibits a different thalamic relay neuron (green, second from bottom) or a thalamic inhibitory neuron (red; RTo). 


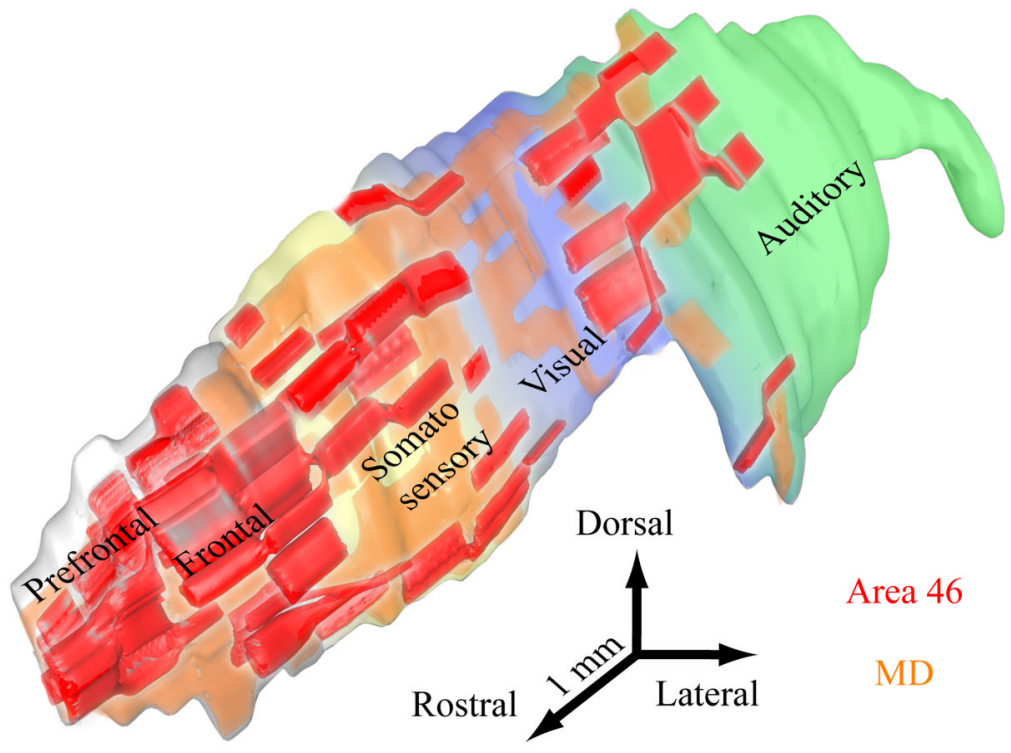

Figure 7.

Cortical and mediodorsal thalamic projections mapped onto the thalamic reticular nucleus in primates. Three-dimensional reconstruction of the thalamic reticular nucleus showing the different sectors of the reticular nucleus based on projections from cortical areas. Projections from prefrontal cortices (red) and the mediodorsal nucleus (MD, orange) in the rhesus monkey are concentrated in the rostral (prefrontal) sector of the reticular nucleus, but also extend to the posterior sectors of the nucleus, including its visual (blue) and auditory sectors (green). 


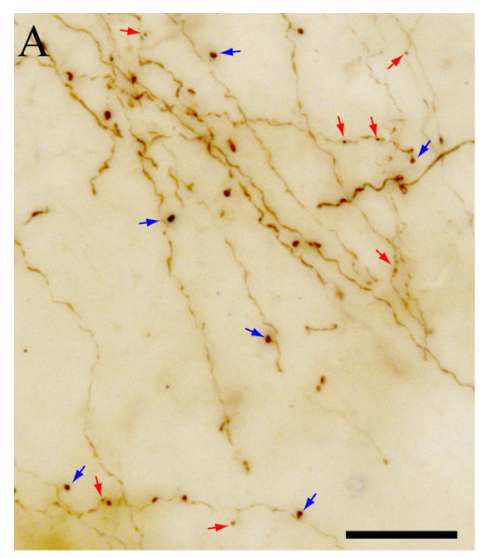

C

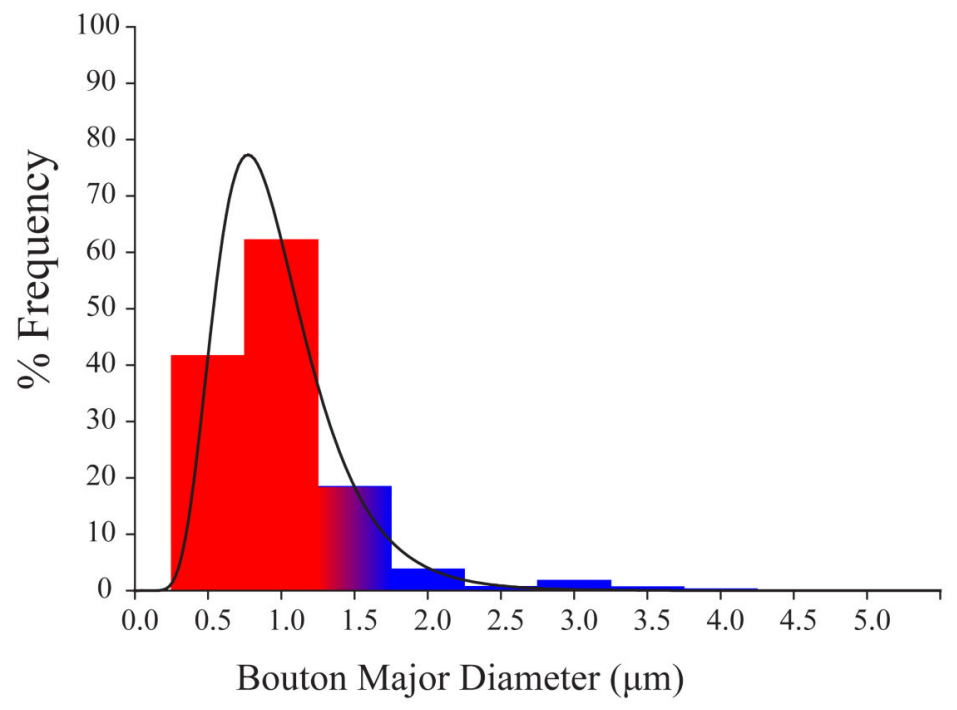

B

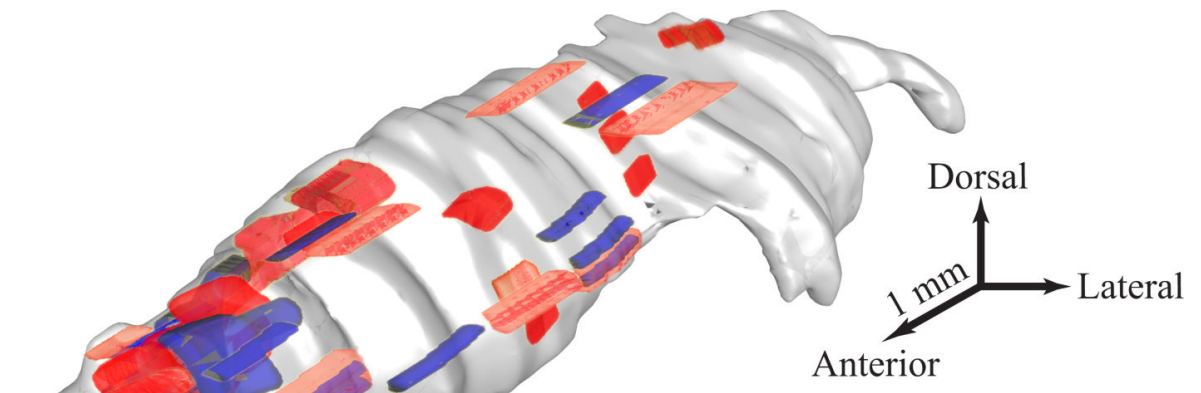

Small boutons

Large boutons

D

Plot of means for each cluster

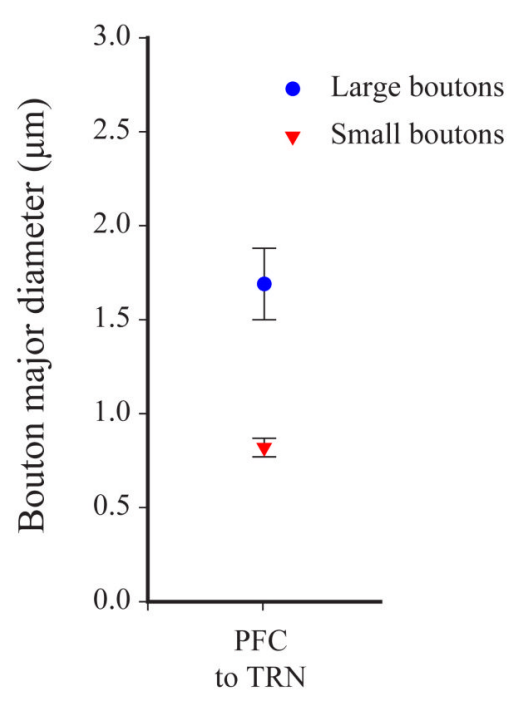

Figure 8.

Prefrontal cortices innervate the thalamic reticular nucleus through small and large terminals. $\boldsymbol{A}$, Brightfield photomicrograph showing small (red arrows) and large (blue arrows) terminals in the reticular nucleus from axons originating in prefrontal cortex of the rhesus monkey. $\boldsymbol{B}$, The distribution of small (red) and large (blue) terminals from prefrontal axons onto the reconstructed reticular nucleus. $\boldsymbol{C}$, Relative frequency of small $(<1.4 \mu \mathrm{m})$ and large $(>1.4$ $\mu \mathrm{m})$ terminals from prefrontal axons in the reticular nucleus. $\boldsymbol{D}$, Cluster analysis shows the presence of a group of small and large axonal boutons from prefrontal cortices (PFC) terminating in the reticular nucleus (TRN). 


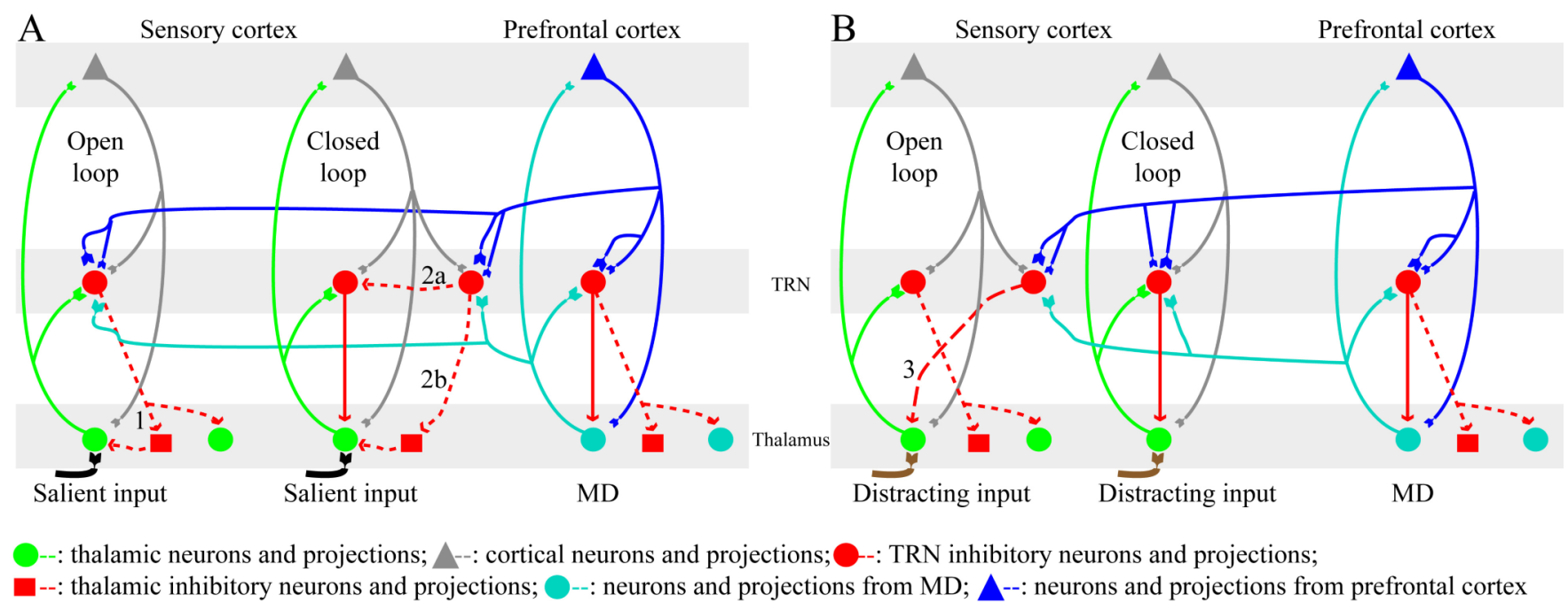

Figure 9.

Schematic diagram summarizing the involvement of sensory and prefrontal systems in attentional mechanisms through the reticular nucleus (TRN). A and B depict possible combinations of salient (black) and distracting (brown) inputs interacting with open or closed reticulothalamic loops. Reticulo-MD loops can be either closed or open. $\boldsymbol{A}$, $\boldsymbol{l e f t}$, open loop circuit: Salient input is relayed from the thalamus to the cortex (green dots and lines) and back to the thalamus (gray triangles and lines); an activated reticular neuron inhibits other thalamic neurons (dotted red lines) or thalamic GABAergic neurons (red squares; 1), allowing prolonged access of the stimulus to the cortex. Sensory cortical or dimorphic prefrontal input (blue) through small and large terminals, and input from MD (cyan) can also activate pathways that disinhibit thalamic neurons relaying relevant information (1). A, right, closed loop circuit: Salient input reaches the cortex briefly before the relay neuron is inhibited by a TRN neuron. Input from sensory or prefrontal cortices and MD can reverse this outcome by activating a neighboring TRN neuron that inhibits the TRN neuron, removing inhibition of the relay neuron (2a), or by inhibiting thalamic GABAergic neurons and disinhibiting the relay neuron (2b). $\boldsymbol{B}$, left, Open loop circuit: Distracting input gains prolonged access to the cortex. Sensory corticoreticular terminations or dimorphic prefrontal input (blue) through small and large terminals, and input from MD (cyan) can activate reticular neurons that inhibit the thalamic relay neuron (3). B, right, closed loop circuit: Distracting input reaches the cortex briefly before the relay neuron is inhibited by a TRN neuron. Input from prefrontal cortices and MD can greatly increase inhibition to the thalamic relay neuron and mask distractors through their numerous small, and some large endings. Some prefrontal and all mediodorsal endings on TRN are large, but sensory projections are exclusively small. 


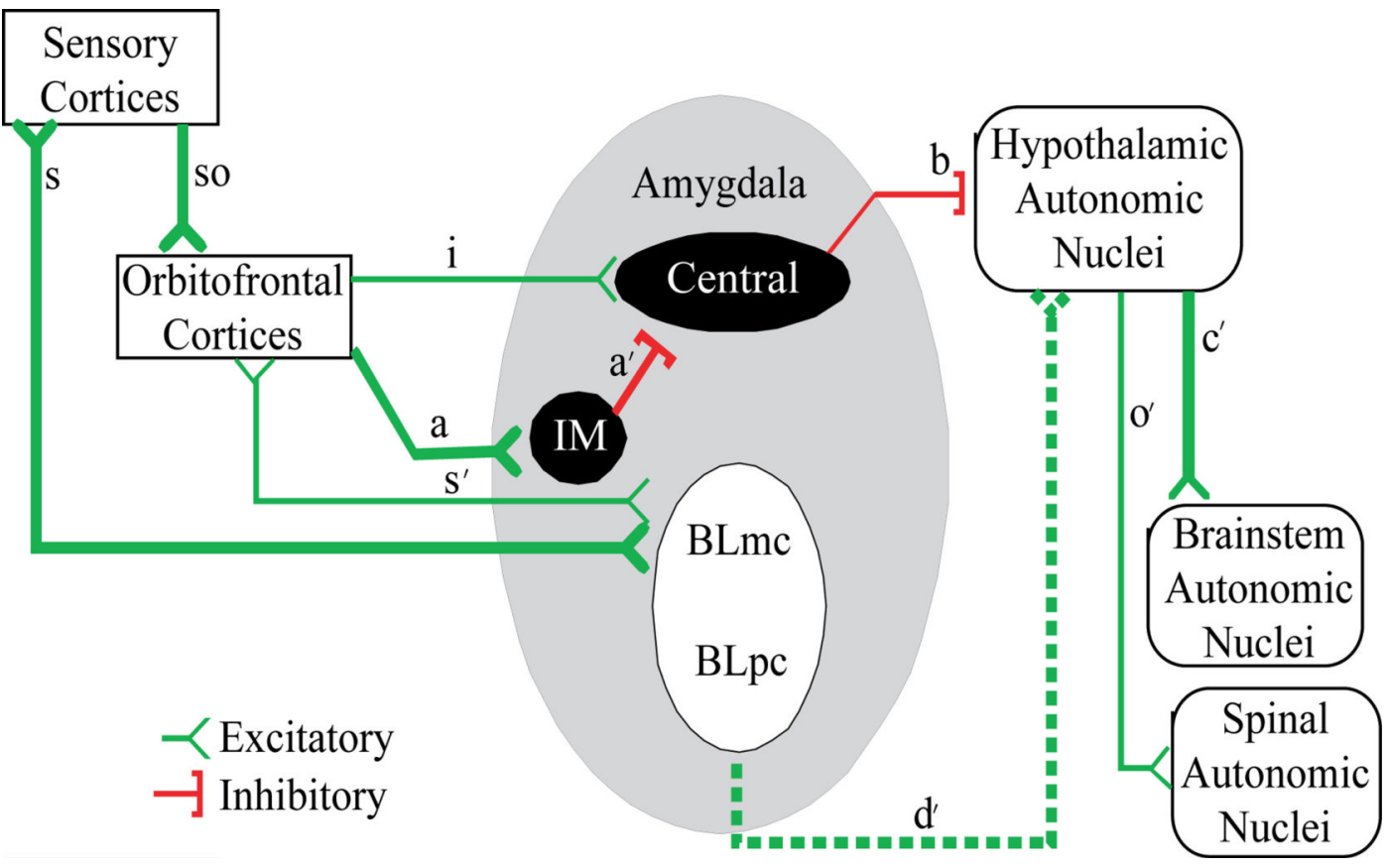

Figure 10.

The orbitofrontal cortex receives widespread connections from sensory cortices (so) and has a special connectional relationship with the amygdala. The posterior orbitofrontal cortex targets heavily the intercalated masses (IM), an inhibitory system in the amygdala. Pathway (a) shows a heavy projection from posterior orbitofrontal cortex to IM, which sends inhibitory projections to the central nucleus of the amygdala $\left(a^{\prime}\right)$, which sends inhibitory projections to hypothalamic autonomic nuclei (b). Activation of the orbitofrontal pathway (a) may then lead to activation of sequential pathways $\left(a, a^{\prime}, b, c^{\prime}, o^{\prime}\right)$, resulting in increased autonomic drive of peripheral autonomic organs (e.g., the lungs and the heart), which are innervated by spinal autonomic nuclei. There is a lighter pathway from orbitofrontal cortex to the central nucleus (i), and its activation would have the opposite effect, resulting in decreased autonomic drive and return to autonomic homeostasis. Adapted from (Barbas and others 2003). 